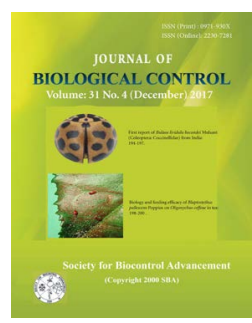

\title{
Host-insect and host-plant associated diversity in microbiota isolated from most important Oriental-Australian region egg parasitoid
}

\author{
S. K. JALALI ${ }^{*}$, S. SRIRAM ${ }^{2}$, T. VENKATESAN ${ }^{1}$, R. P. MORE ${ }^{1}$, OMPRAKASH NAVIK ${ }^{1}$, Y. LALITHA ${ }^{1}$ and \\ RAKSHIT OJHA ${ }^{1}$ \\ ${ }^{1}$ ICAR-National Bureau of Agricultural Insect Resources, Post Bag No. 2491, H. A. Farm Post, Bellary Road, Hebbal, Bangalore - 24, \\ Karnataka, India \\ ${ }^{2}$ ICAR-Indian Institute of Horticultural Research, Hessaraghatta Lake Post, Bangalore - 89, Karnataka, India \\ ${ }^{*}$ Corresponding author E-mail: jalalisk1910@yahoo.co.in
}

\begin{abstract}
Host-insect and host-plant associated differentiation of genetically divergent microbiota were recorded from economically important egg parasitoid collected from 26 locations in India, Trichogramma chilonis constituted 86.8\% of the populations collected. It was recorded from 14 host-insects, 14 different crops and weed plants from 12 states. Nine species of yeast were recorded from parasitoid from 5 host-insects with Wickerhamomyces anamalus was isolated from $36.4 \%$ samples and highest numbers were recorded from parasitoid collected on sugarcane. Bacillus cereus, Pseudomonas sp. and Stenotrophomonas maltophilia from T. chilonis constitute $64.3 \%$ of bacterial diversity based on their $16 \mathrm{~S}$ rDNA sequences. For taxonomic identification using 16s rDNA and ITS sequences, we performed taxonomic classification of total 33 ITS isolates against UNITE Fungal ITS database and assigned taxonomy hierarchy to the sequences. Also, a total of 13 isolates 16s rDNA sequences were taxonomically assigned against RDP 16s rDNA database using RDP Naive Bayesian rRNA Classifier Version 2.1. Most of the species are correctly identified in the respective species members with high confidence threshold value support.
\end{abstract}

KEY WORDS: Egg parasitoid, evolutionary relationship, host-insects, host-plants, microbiota

(Article chronicle: Received: 23-09-2017; Revised: 12-11-2017; Accepted: 10-12-2017)

\section{INTRODUCTION}

The insect gut is known to harbor diverse microbiota that often perform beneficial role, though some are known to have opportunistically harmful role (Bignell, 1984; Kaufman and Klug, 1991; Dillon and Dillon, 2004; Bernays and Klein, 2002; Tagami et al., 2006; Bourtzis and Miller, 2003; Broderick et al., 2006). Several yeasts have been recorded in insects, mainly those feeding on fruiting bodies, flowers and nectar (Lachance and Bowles, 2002; Suh et al., 2004; Nguyen et al., 2006). Ferrari et al., (2006) reported host-associated differentiation in divergent microbiota isolated from pea aphid. Host-Insect and host-plant associated divergence was proposed as a mechanism promoting greater than before species range (Stireman et al., 2005). It has been proposed that the microbiota diversity of insects play a crucial role in host-insect or host-plant as- sociated diversity by allowing insects to use different plant species (Ferrari and Vavre, 2011; Gauthier et al., 2015). The microbiota of the insects has been quantified using culturedependent and culture-independent methods (Mrázek et al., 2008; Chandler et al., 2011; Toju and Fukatsu, 2011; Ngo et al., 2015; Montagna et al., 2015). Culture-dependent methods often produce results based on microbes that can be cultured on artificial media. In metagenomics approach, culture-independent molecular analyses of the ITS gene for yeast species and 16S rRNA gene for bacteria has produced a enhanced and more complete representation of communities of insects. The latest advances in the molecular biological use through next generation sequencing technologies have resulted in quantification of the significantly larger number of microbiota than previously estimated by traditional culture-based method (Yun et al., 2014). The analysis of microbial diversity with its link to host insect and host- 
plant is vital for a better understanding of the ecological aspects. However, most studies carried out so far focused on insect pests or predators, but none of them have been on parasitoids like Trichogramma, except for work done by Srinatha et al., (2015).

The relationship of insect populations with various host-plants may depend on suitable microbiota harbored by them, in such cases there is the possibility of a divergence of various species and or strains (Medina et al., 2011). Bilodeau et al., (2013) reported that insect parasitoids and their host-insects represent a wide range of parasitic trophic relationship and observed a strong variation of the predictive power of intrinsic (body color) and extrinsic traits (symbionts, host plant). Host variables considered as key predictors of outcomes strongly interact and cannot be considered in isolation.

Among various natural enemies used for suppression of insect pests, egg parasitoids trichogrammatids are the most widely used, particularly for lepidopteran pests on several crops such as rice, corn, sugarcane, cotton, several vegetable and fruit crops, and forest trees, covering more than 33 crops and 52 insect species (Hassan, 1997) because of their ability to manage several insect pests and are easily mass-produced. Trichogramma chilonis Ishii is the most widely used species in an integrated pest management programme in South, Southeast Asia, Far-east, Indo-China region and Pacific region. It has also been reported as exotic in Kenya, Spain, South Africa and Australia (Jalali et al., 2006). It is often cited that compared to the laboratory-bred population, field-collected populations of Trichogramma are robust and have a higher biological fitness (Nagarkatti and Nagaraja, 1978; Jalali and Singh, 1993).

In the present work, we investigated the association of microbiota isolated from egg parasitoids collected from fourteen crops including weed plants. There is no work done to date on the diversity and composition of microbes associated with trichogrammatids and corresponding hostplants. The present study, therefore, aimed to investigate the diversity of the microbiota through extensive sampling approach and sequencing technique.

\section{MATERIALS AND METHODS}

\section{Insect collection}

At least fifty eggs and about 20 eggs-masses of insects were collected from 14 insect species, including unknown insect eggs on 14 crops, including weed plants, 12 different states covering 26 locations in India, ranging from GPS coordinates of $11.018^{\circ}$ to $34.090^{\circ} \mathrm{N}$ and $73.200^{\circ}$ to $94.220^{\circ} \mathrm{E}$ (Table 1). These eggs were brought to Molecular Entomol- ogy laboratory, ICAR-NBAIR, Bangalore, for observing the emergence of the egg parasitoids. After emergence, the parasitoids were identified by a renowned Trichogramma taxonomist, Dr. H. Nagaraja, for their identity and were immediately used for isolation of microbiota.

Isolation of endosymbionts from different Trichogramma species collected from various locations, host-insects and crops

Adult wasps of Trichogramma were released in sterile Eppendorf vials $(1.5 \mathrm{ml})$ and were frozen at $-70^{\circ} \mathrm{C}$ deep freezer (M/s New Brunswick Ultra Low Freezer CFC free Model U41085). Such frozen wasps were allowed to thaw for $10 \mathrm{~min}$ and were first washed with $70 \%$ ethanol for 1 min followed by surface sterilization with $10 \%$ sodium hypochlorite for 5 minutes to remove the adhering contaminants especially the external microflora and washed with distilled water five times (Meyer and Hoy, 2008). This method of surface sterilization prior to extraction of microbiota is standard as endorsed by Hammer et al., (2015), who used five different methods such as freezing, ethanol, Dimethyl Sulfoxide (DMSO), Cetrimonium Bromide (CTAB), and room-temperature storage without preservative and found that storage method had little to no effect on assessments of microbiota composition. Five to 10 Trichogramma adults were transferred into sterile Eppendorf vials $(1.5 \mathrm{ml})$, using a camel hair brush ( 0 number) and were homogenized with a micro-pestle in $50 \mu$ l sterile distilled water in a Laminar Flow Chamber (M/s Alpha Linear, Bangalore, India). $50 \mu \mathrm{l}$ of the supernatant was pipetted out on Yeast Extract Peptone, Dextrose (YEPD) agar amended with and without chloramphenicol/cycloheximide@100ppm in each plate and spread using a L shaped spreader and the plates were incubated for 48 hours in a BOD incubator (M/s Remi Instruments, Bangalore, India) maintained at $26 \pm 0.5^{\circ} \mathrm{C}$ and $60 \pm 2 \%$ RH.

\section{DNA isolation, amplification, sequencing, and identifi- cation}

\section{Yeast DNA isolation and amplification}

Yeast colonies obtained on chloramphenicol amended YEPD medium were sub cultured on yeast extract peptone, dextrose broth and incubated for 48 hours in a BOD incubator maintained at $26 \pm 0.5^{\circ} \mathrm{C}$ and $60 \pm 2 \% \mathrm{RH}$ for $48 \mathrm{~h}$. One $\mathrm{ml}$ of the cell suspension was transferred to a fresh $1.5 \mathrm{ml}$ eppendorf tube and centrifuged at $7000 \mathrm{rpm}$ for 3 minutes. The cells were re-suspended in sorbitol buffer. Genomic DNA was extracted using bacterial and yeast genomic DNA miniprep purification kit (M/s Himedia Laboratories, India) as per manufacturer's instructions. The extracted DNA was stored at $-20^{\circ} \mathrm{C}$ for further use. Each population of yeast was maintained individually. 
The fungus-specific universal primers ITS1 and ITS4 were used to amplify the ITS region as described by White et al., (1990). The primers used for YITS-PCR: YITS Forward: 5' TCCGTAGGTGAACCTGCGG3' YITS Reverse: 5'TCCTCCGCTTATTGATATGC3'. PCR was performed in a total volume of $50 \mu \mathrm{l}$ consisting of $10 \mathrm{X}$ Taq buffer, $10 \mathrm{mM}$ dNTP's, $1 \mathrm{U}$ of Taq DNA polymerase, 20 pmol each

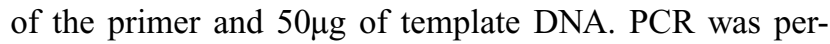
formed by employing the following conditions: samples were subjected to $94^{\circ} \mathrm{C}$ for initial denaturation for $3 \mathrm{~min}$ utes; followed by 30 cycles of denaturation at $94^{\circ} \mathrm{C}$ for 1 min, annealing at $60^{\circ} \mathrm{C}$ for 1 minute, and extension at $72^{\circ} \mathrm{C}$ for 1 minute and extended elongation step was performed at $72^{\circ} \mathrm{C}$ for $3 \mathrm{~min}$. With each run a negative control was performed with nuclease free water in place of template DNA in the PCR mixture.

\section{Bacterial DNA isolation and amplification}

Similar to yeast cultures, bacterial colonies obtained on cycloheximide amended medium were sub cultured on Nutrient Agar consisting of (mass/volume): $0.5 \%$ Peptone, $0.3 \%$ yeast extract, $1.5 \%$ agar, $0.5 \%$ Nacl and distilled water and finally $\mathrm{pH}$ is adjusted to neutral. For DNA extraction, the cultures were grown on a nutrient broth for 48 hours. The primers used for 16S rDNA: 16S Forward: 5'AGAGTTTGATCCTGGCTCAG 3' and 16S Reverse: 5'CGGTGTGTACAAGACCC 3'. PCR was performed in a total volume of $50 \mu \mathrm{l}$ consisting of $10 \mathrm{X}$ Taq buffer, $15 \mathrm{mM}$ $\mathrm{MgCl}_{2}, 10 \mathrm{mM}$ dNTP's, $1 \mathrm{U}$ of Taq DNA polymerase (M/s MBI Fermentas, Germany), 20 pmol each of the 16S rDNA

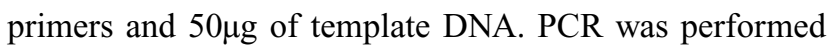
by employing the following conditions: samples were subjected to $94^{\circ} \mathrm{C}$ for initial denaturation for $3 \mathrm{~min}$; followed by 30 cycles of denaturation at $94^{\circ} \mathrm{C}$ for 1 minute, annealing at $60^{\circ} \mathrm{C}$ for 1 minute, and extension at $72^{\circ} \mathrm{C}$ for 1 minute and extended elongation step was performed at $72^{\circ} \mathrm{C}$ for $3 \mathrm{~min}$ utes. With each run a negative control was performed with nuclease free water in place of template DNA in the PCR mixture. PCR products were electrophoresed on a 1.8\% low melting point agarose gel (M/s Genei, Bangalore, India). Gels were stained using ethidium bromide @ $0.5 \mu \mathrm{g} / \mathrm{ml}$. A $100 \mathrm{bp}$ molecular weight marker (M/s MBI Fermentas, Germany) was run along with the samples for reference.

\section{DNA isolation and Wolbachia amplification}

The primers used for wsp and ftsZ were as follows: Initially the general primer 81F-5' TGGTCCAAGTGATGAAGAAAC 3' and 691R- 5' AAAAATTAAACGCTACTCCA 3 ' was used to know the presence of Wolbachia endosymbiont and then screened with the group-specific ftsz-A and ftsZ-B: 308F- 5' TTAAAGATGTAACATTTG 3' and 691R-
5' AAAAATTAAACGCTACTCCA 3'. The B group gene primer used was 183F-5' AAGGAACCGAAGTTCATG 3' and 691R- 5' AAAAATTAAACGCTACTCCA 3'. PCR was performed in a total reaction volume of $50 \mu \mathrm{l}$ consisting of 10X Taq buffer, $15 \mathrm{mM} \mathrm{MgCl}_{2}, 10 \mathrm{mM}$ dNTP's, $1 \mathrm{U}$ of Taq DNA polymerase (M/s MBI Fermentas, Germany), 20 pmol each of the wsp and ftsZ-A and ftsZ-B primers and $50 \mu \mathrm{g}$ of template DNA. PCR was performed by employing the following conditions: samples were subjected to $94^{\circ} \mathrm{C}$ for initial denaturation for 3 minutes; followed by 30 cycles of denaturation at $94^{\circ} \mathrm{C}$ for 1 minute, annealing at $55-60^{\circ} \mathrm{C}$ for $1 \mathrm{~min}$, and extension at $72^{\circ} \mathrm{C}$ for 1 minute and extended elongation step was performed at $72^{\circ} \mathrm{C}$ for 3 minutes. With each run a negative control was performed with nuclease free water in place of template DNA in the PCR mixture. PCR products were electrophoresed on a $1.8 \%$ low melting point agarose gel (M/s Genei, Bangalore). Gels were stained using ethidium bromide @ $0.5 \mu \mathrm{g} / \mathrm{ml}$. A 100 bp molecular weight marker (M/s MBI Fermentas, Germany) was run along with the samples for reference.

\section{Yeast and bacterial DNA sequencing and identification}

The amplification products were purified with the QIA-Quick PCR purification kit (M/s Qiagen, Hilden, Germany). DNA fragments were extracted from the gel using the Qia Quick Gel extraction kit (M/s Qiagen, Hilden, Germany). The DNA fragments were sequenced using an $\mathrm{ABI}$ prism 310 DNA sequencer using Big Dye Terminator reaction. Yeast and bacterial species were identified by searching databases using the BLAST sequence analysis tool. The ITS1-5.8S-ITS2 region and 16s rDNA sequences were compared with sequences acquired from GenBank ITS and 16s rDNA sequences for yeast and bacteria, respectively, using nucleotide BLAST (Blastn). Species identification was determined from the lowest E-value of the BLAST output.

Taxonomic classification of endosymbionts cultured from different populations of Trichogramma using RDP classifier

Our 13 Bacterial 16S rDNA sequence classification was performed against the RDP 16S rRNA reference database (release accessed $7^{\text {th }}$ October 2016) in RDP Naive Bayesian rRNA Classifier Version 2.11 (http://rdp.cme. msu.edu/classifier/classifier.jsp) (Wang et al., 2007) and all 33 isolates of fungal ITS sequences were searched against UNITE Fungal ITS database (trainset 07/04/2014) using RDP Naive Bayesian rRNA Classifier Version 2.1 (Deshpande et al., 2016). We used procedural tutorials for RDP Classifier to analyse 16S rDNA and ITS sequences. Taxonomic hierarchical classification is done with confidence threshold $80 \%$. 


\section{RESULTS AND DISCUSSION}

Out of 53 Trichogramma specimens obtained from 26 locations across 12 states, 46 were identified as T. chilonis Ishii, 3 as T. achaeae Nagaraja and Nagarkatti and 4 as $T$. danausicida Nagaraja (Table 1). Among three species, $T$. chilonis was recorded from eight host-insect eggs in nine crops and, T. achaeae from three host-insects on two crops and T. danausucida from 4 unknown host-insect eggs on weed plants. Trichogramma chilonis was found to occur more frequently from all the states from which collections were made, however, T. achaeae and T. danausicida was recorded from 2 and 1 states, respectively. These were purified by singe colony isolation by repeating the process thrice. Wolbachia was not amplified from any of 53 samples in the present study by two primers used, indicating its absence in samples collected from different places in India.

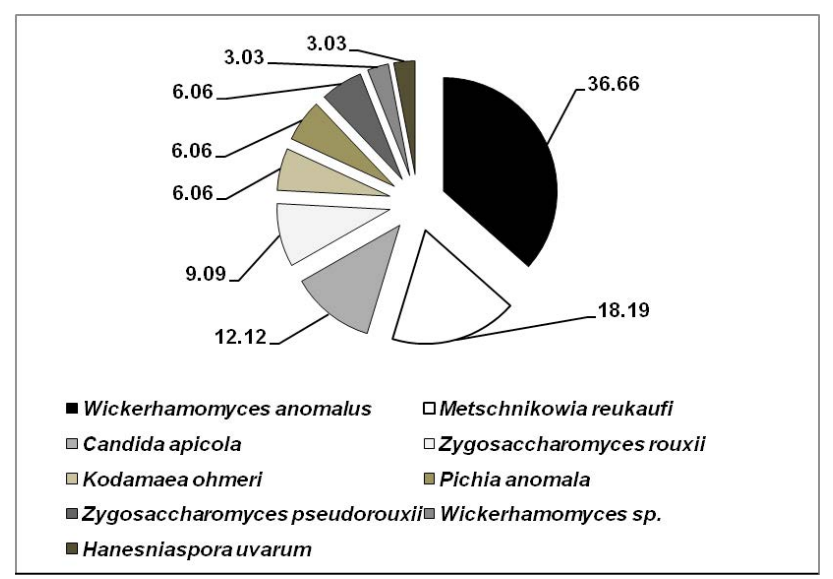

(A)

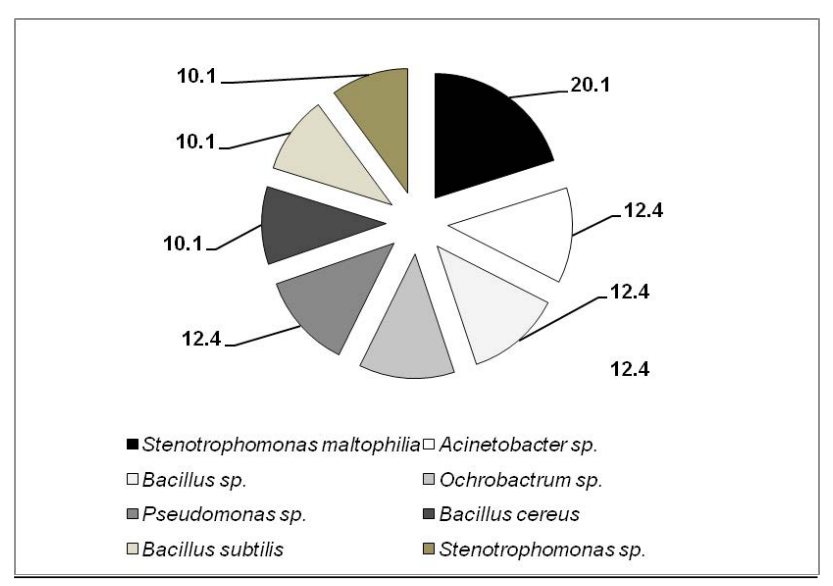

(B)

Fig. 1. Diversity of yeast (A) and bacteria (B) microbiota recorded from Trichogramma spp.

Nine species of yeast were recorded from 5 host insects, Wickerhamomyces anomalus was recorded from $36.4 \%$, Metschnikowia reukaufii from $18.2 \%$, Candida api- cola from $12.1 \%$ samples and Zygosaccharomyces rouxii from $9.1 \%$ samples, constituting $75.8 \%$ of the yeast diversity based on their ITS sequences (Fig. 1). Highest yeast species were recorded from parasitoid collected from sugarcane ( 7 species) and five species from tomato and other 4 host-plant contributed 1 each (Table 2). Eight bacterial species were recorded from three crops and from 4 states. Bacillus cereus was recorded from $28.6 \%$, Pseudomonas from 21.4\% and Stenotrophomonas maltophilia from $14.3 \%$ samples, while other species like Acinetobacter sp., Bacillus sp., Bacillus subtilis, Ochrobactrum sp., Stenotrophomonas sp. were recorded from $7.14 \%$ samples based on their 16S rDNA sequences (Table 1). Wickerhamomyces anomalus was recorded from $23.5 \%$ of the host-plants and from $57.1 \%$ of host-insects, thus making it the most abundant yeast species and other important species was Metschnikowia reukaufii, which was recorded from 17.6 and $28.6 \%$ of host-plants and host-insects, respectively. In contrast, bacterial species were observed consistently across host-plants and host-insects (Fig. 1 and 2).

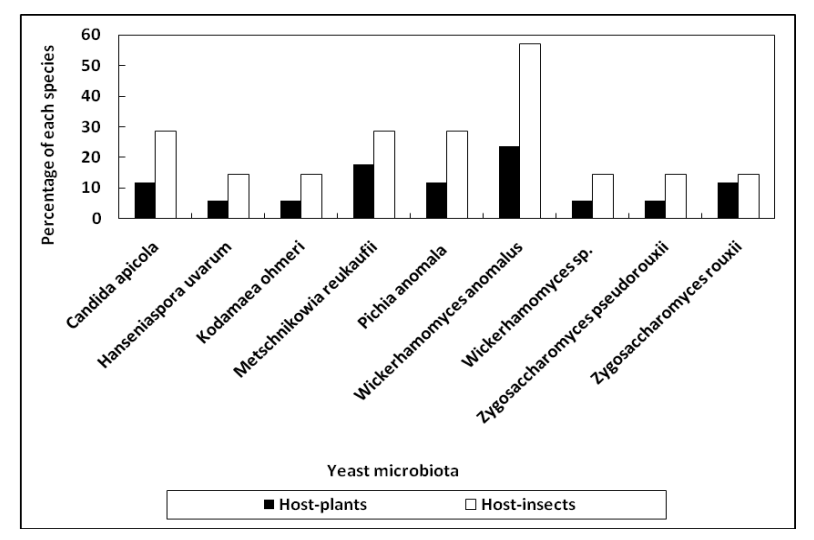

(A)

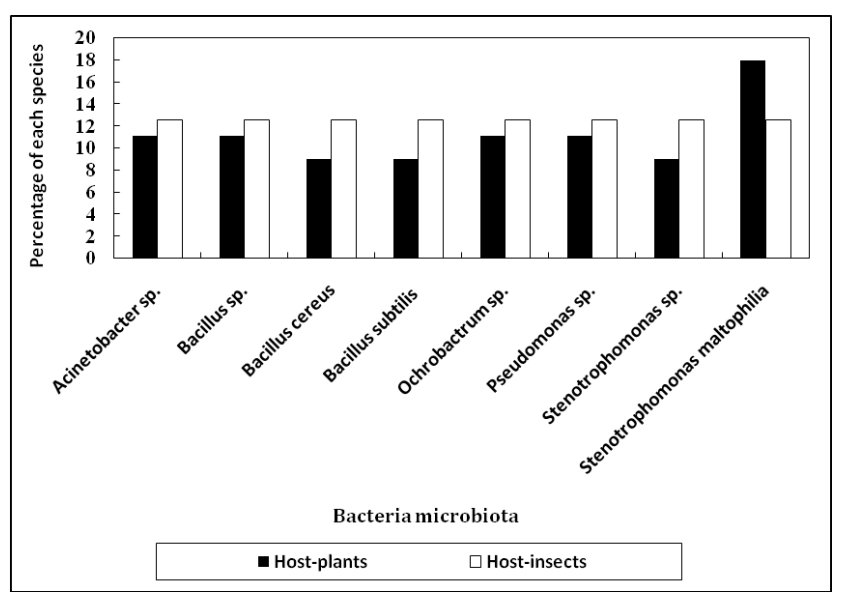

(B)

Fig. 2. Percentage composition of all yeast (A) and bacteria (B) on different host-plants and different host-insects. 
Table 1. List of locations, host-plant and host-insects and populations of Trichogramma collected in India, microorganisms isolated from them and GenBank accession numbers of ITS and 16s rDNA sequences of yeasts and bacteria, respectively

\begin{tabular}{|c|c|c|c|c|c|c|c|c|}
\hline $\begin{array}{l}\text { L. } \\
\text { No. }\end{array}$ & Species & Host-plants & Host-Insects & Location & State & $\begin{array}{l}\text { GPS Coor- } \\
\text { dinates }\end{array}$ & Organism & $\begin{array}{l}\text { GenBank } \\
\text { accn. no. }\end{array}$ \\
\hline \multicolumn{9}{|c|}{ Cultivable yeast isolated from Trichogramma } \\
\hline \multirow[t]{2}{*}{1} & \multirow[t]{2}{*}{ T. chilonis } & \multirow[t]{2}{*}{ Tomato } & \multirow[t]{2}{*}{$\begin{array}{l}\text { Helicoverpa } \\
\text { armigera }\end{array}$} & \multirow[t]{2}{*}{ Coimbatore } & \multirow[t]{2}{*}{ Tamil Nadu } & \multirow[t]{2}{*}{$\begin{array}{l}11.018^{\circ} \mathrm{N} \\
76.973^{\circ} \mathrm{E}\end{array}$} & $\begin{array}{l}\text { Zygosaccharomy- } \\
\text { cespseudorouxii }\end{array}$ & JF803489 \\
\hline & & & & & & & $\begin{array}{l}\text { Zygosaccharomy- } \\
\text { cespseudorouxii }\end{array}$ & JF803490 \\
\hline \multirow[t]{2}{*}{2} & \multirow{2}{*}{ T. chilonis } & \multirow{2}{*}{ Sugarcane } & \multirow{2}{*}{$\begin{array}{l}\text { Chilo infuscatel- } \\
\text { lus }\end{array}$} & \multirow[t]{2}{*}{ Cuddalore } & \multirow[t]{2}{*}{ Tamil Nadu } & \multirow{2}{*}{$\begin{array}{l}11.750^{\circ} \mathrm{N} \\
79.750^{\circ} \mathrm{E}\end{array}$} & Kodamaea ohmeri & HQ696999 \\
\hline & & & & & & & $\begin{array}{l}\text { Zygosaccharomy- } \\
\text { ces rouxii }\end{array}$ & HQ696996 \\
\hline 3 & T. chilonis & Sugarcane & $\begin{array}{l}\text { Chilo infuscatel- } \\
\text { lus }\end{array}$ & Mandya & Karnataka & $\begin{array}{l}12.520^{\circ} \mathrm{N} \\
76.900^{\circ} \mathrm{E}\end{array}$ & Kodamaea ohmeri & HQ696998 \\
\hline \multirow[t]{7}{*}{4} & \multirow[t]{2}{*}{ T. chilonis } & \multirow[t]{2}{*}{ Tomato } & \multirow[t]{2}{*}{$\begin{array}{l}\text { Helicoverpa } \\
\text { armigera }\end{array}$} & \multirow[t]{7}{*}{ Bangalore } & \multirow[t]{7}{*}{ Karnataka } & \multirow[t]{7}{*}{$\begin{array}{l}12.967^{\circ} \mathrm{N} \\
77.567^{\circ} \mathrm{E}\end{array}$} & $\begin{array}{l}\text { Wickerhamomyces } \\
\text { anomalus }\end{array}$ & JF416789 \\
\hline & & & & & & & $\begin{array}{l}\text { Zygosaccharomy- } \\
\text { ces rouxii }\end{array}$ & JF416790 \\
\hline & T. achaeae & Sun hemp & Unknown eggs & & & & $\begin{array}{l}\text { Wickerhamomyces } \\
\text { anomalus }\end{array}$ & JF728870 \\
\hline & T. chilonis & Rose & $\begin{array}{l}\text { Helicoverpa } \\
\text { armigera }\end{array}$ & & & & Pichia anomala & FJ599744 \\
\hline & \multirow[t]{3}{*}{$\begin{array}{l}\text { T. danau- } \\
\text { sicida }\end{array}$} & \multirow[t]{3}{*}{ Weed } & \multirow[t]{3}{*}{ Unknown eggs } & & & & $\begin{array}{l}\text { Wickerhamomyces } \\
\text { anomalus }\end{array}$ & JN210893 \\
\hline & & & & & & & $\begin{array}{l}\text { Wickerhamomyces } \\
\text { anomalus }\end{array}$ & JN210892 \\
\hline & & & & & & & $\begin{array}{l}\text { Wickerhamomyces } \\
\text { anomalus }\end{array}$ & JF803491 \\
\hline 5 & T. chilonis & Tomato & $\begin{array}{l}\text { Helicoverpa } \\
\text { armigera }\end{array}$ & Malur & Karnataka & $\begin{array}{l}13.021^{\circ} \mathrm{N} \\
77.938^{\circ} \mathrm{E}\end{array}$ & $\begin{array}{l}\text { Metschnikowia } \\
\text { reukaufii }\end{array}$ & HM222406 \\
\hline 6 & T. chilonis & Tomato & $\begin{array}{l}\text { Helicoverpa } \\
\text { armigera }\end{array}$ & Kolar & Karnataka & $\begin{array}{l}13.133^{\circ} \mathrm{N} \\
78.133^{\circ} \mathrm{E}\end{array}$ & $\begin{array}{l}\text { Metschnikowia } \\
\text { reukaufii }\end{array}$ & HM222405 \\
\hline 7 & T. chilonis & Tomato & $\begin{array}{l}\text { Helicoverpa } \\
\text { armigera }\end{array}$ & Rajankunte & Karnataka & $\begin{array}{l}13.181^{\circ} \mathrm{N} \\
77.554^{\circ} \mathrm{E}\end{array}$ & $\begin{array}{l}\text { Metschnikowia } \\
\text { reukaufii }\end{array}$ & HM222408 \\
\hline 8 & T. chilonis & Tomato & $\begin{array}{l}\text { Helicoverpa } \\
\text { armigera }\end{array}$ & $\begin{array}{l}\text { Doddaballa- } \\
\text { pur }\end{array}$ & Karnataka & $\begin{array}{l}13.292^{\circ} \mathrm{N} \\
77.543^{\circ} \mathrm{E}\end{array}$ & $\begin{array}{l}\text { Metschnikowia } \\
\text { reukaufii }\end{array}$ & HM222407 \\
\hline 9 & T. chilonis & Tomato & $\begin{array}{l}\text { Helicoverpa } \\
\text { armigera }\end{array}$ & Vikarabad & Telangana & $\begin{array}{l}17.330^{\circ} \mathrm{N} \\
77.900^{\circ} \mathrm{E}\end{array}$ & $\begin{array}{l}\text { Wickerhamomyces } \\
\text { anomalus }\end{array}$ & HM601458 \\
\hline 10 & T. chilonis & Tomato & $\begin{array}{l}\text { Helicoverpa } \\
\text { armigera }\end{array}$ & Hyderabad & Telangana & $\begin{array}{l}17.370^{\circ} \mathrm{N} \\
78.480^{\circ} \mathrm{E}\end{array}$ & Candida apicola & FJ713025 \\
\hline 11 & T. chilonis & Sugarcane & $\begin{array}{l}\text { Chilo infuscatel- } \\
\text { lus }\end{array}$ & Anakapalli & $\begin{array}{l}\text { Andhra } \\
\text { Pradesh }\end{array}$ & $\begin{array}{l}17.680^{\circ} \mathrm{N} \\
83.020^{\circ} \mathrm{E} \\
\end{array}$ & $\begin{array}{l}\text { Wickerhamomyces } \\
\text { sp. }\end{array}$ & HQ696994 \\
\hline \multirow[t]{2}{*}{12} & T. chilonis & Sugarcane & $\begin{array}{l}\text { Chilo infuscatel- } \\
\text { lus }\end{array}$ & \multirow[t]{2}{*}{ Pune } & \multirow[t]{2}{*}{ Maharashtra } & \multirow[t]{2}{*}{$\begin{array}{l}18.520^{\circ} \mathrm{N} \\
73.857^{\circ} \mathrm{E}\end{array}$} & $\begin{array}{l}\text { Wickerhamomyces } \\
\text { anomalus }\end{array}$ & HQ696991 \\
\hline & T. achaeae & Sun hemp & Unknown eggs & & & & Pichia anomala & FJ224365 \\
\hline 13 & T. chilonis & Sugarcane & $\begin{array}{l}\text { Chilo infuscatel- } \\
\text { lus }\end{array}$ & $\begin{array}{l}\text { Bhubanesh- } \\
\text { war }\end{array}$ & Odisha & $\begin{array}{l}20.270^{\circ} \mathrm{N} \\
85.840^{\circ} \mathrm{E}\end{array}$ & $\begin{array}{l}\text { Wickerhamomyces } \\
\text { anomalus }\end{array}$ & HQ696992 \\
\hline \multirow[t]{2}{*}{14} & T. chilonis & Sugarcane & $\begin{array}{l}\text { Chilo infuscatel- } \\
\text { lus }\end{array}$ & Navasari & Gujarat & $\begin{array}{l}20.949^{\circ} \mathrm{N} \\
72.914^{\circ} \mathrm{E}\end{array}$ & $\begin{array}{l}\text { Wickerhamomyces } \\
\text { anomalus }\end{array}$ & HQ615929 \\
\hline & & & & & & & $\begin{array}{l}\text { Zygosaccharomy- } \\
\text { ces rouxii }\end{array}$ & HQ696997 \\
\hline 15 & T. chilonis & Sesame & Unknown eggs & Amreli & Gujarat & $\begin{array}{l}21.620^{\circ} \mathrm{N} \\
71.230^{\circ} \mathrm{E} \\
\end{array}$ & Pichia anomala & FJ224365 \\
\hline 16 & T. chilonis & Sugarcane & $\begin{array}{l}\text { Chilo infuscatel- } \\
\text { lus }\end{array}$ & Vadodara & Gujarat & $\begin{array}{l}22.300^{\circ} \mathrm{N} \\
73.200^{\circ} \mathrm{E} \\
\end{array}$ & $\begin{array}{l}\text { Hanseniaspora } \\
\text { uvarum }\end{array}$ & HM601459 \\
\hline 17 & T. chilonis & Sugarcane & $\begin{array}{l}\text { Chilo infuscatel- } \\
\text { lus }\end{array}$ & Lucknow & $\begin{array}{l}\text { Uttar } \\
\text { Pradesh }\end{array}$ & $\begin{array}{l}26.800^{\circ} \mathrm{N} \\
80.900^{\circ} \mathrm{E}\end{array}$ & $\begin{array}{l}\text { Candida cf. api- } \\
\text { cola }\end{array}$ & HQ697001 \\
\hline & T. chilonis & Sugarcane & Chilo auricilius & & & & $\begin{array}{l}\text { Wickerhamomyces } \\
\text { anomalus }\end{array}$ & HQ615930 \\
\hline
\end{tabular}




\begin{tabular}{|c|c|c|c|c|c|c|c|c|}
\hline \multirow[t]{2}{*}{18} & \multirow[t]{2}{*}{ T. chilonis } & \multirow[t]{2}{*}{ Sugarcane } & \multirow[t]{2}{*}{ Chilo auricilius } & \multirow[t]{2}{*}{$\begin{array}{l}\text { Shahjahan- } \\
\text { pur }\end{array}$} & \multirow[t]{2}{*}{$\begin{array}{l}\text { Uttar } \\
\text { Pradesh }\end{array}$} & \multirow[t]{2}{*}{$\begin{array}{l}28.000^{\circ} \mathrm{N} \\
79.833^{\circ} \mathrm{E}\end{array}$} & $\begin{array}{l}\text { Candida cf. api- } \\
\text { cola }\end{array}$ & HQ697000 \\
\hline & & & & & & & $\begin{array}{l}\text { Metschnikowia } \\
\text { reukaufii }\end{array}$ & HM601457 \\
\hline 19 & T. chilonis & Sugarcane & $\begin{array}{l}\text { Chilo infuscatel- } \\
\text { lus }\end{array}$ & Uchani & Haryana & $\begin{array}{l}29.690^{\circ} \mathrm{N} \\
76.980^{\circ} \mathrm{E}\end{array}$ & $\begin{array}{l}\text { Candida cf. api- } \\
\text { cola }\end{array}$ & HQ615928 \\
\hline 20 & T. chilonis & Sugarcane & Chilo auricilius & $\begin{array}{l}\text { Nawansha- } \\
\text { har }\end{array}$ & Punjab & $\begin{array}{l}31.125^{\circ} \mathrm{N} \\
76.116^{\circ} \mathrm{E}\end{array}$ & $\begin{array}{l}\text { Wickerhamomyces } \\
\text { anomalus }\end{array}$ & HQ696995 \\
\hline 21 & T. chilonis & Sugarcane & Chilo auricilius & Jalandhar & Punjab & $\begin{array}{l}31.326^{\circ} \mathrm{N} \\
75.576^{\circ} \mathrm{E}\end{array}$ & $\begin{array}{l}\text { Wickerhamomyces } \\
\text { anomalus }\end{array}$ & HQ696993 \\
\hline 22 & T. chilonis & Maize & Chilo partellus & Srinagar & $\begin{array}{l}\text { Jammu \& } \\
\text { Kashmir }\end{array}$ & $\begin{array}{l}34.090^{\circ} \mathrm{N} \\
74.790^{\circ} \mathrm{E}\end{array}$ & $\begin{array}{l}\text { Metschnikowia } \\
\text { reukaufii }\end{array}$ & HQ221884 \\
\hline \multicolumn{9}{|c|}{ Cultivable bacteria isolated from Trichogramma } \\
\hline \multirow[t]{8}{*}{23} & T. chilonis & Cabbage & $\begin{array}{l}\text { Plutella xylo- } \\
\text { stella }\end{array}$ & \multirow[t]{8}{*}{ Bangalore } & \multirow[t]{8}{*}{ Karnataka } & \multirow[t]{8}{*}{$\begin{array}{l}12.967^{\circ} \mathrm{N} \\
77.567^{\circ} \mathrm{E}\end{array}$} & Bacillus cereus & JF728871 \\
\hline & \multirow[t]{7}{*}{ T. chilonis } & \multirow[t]{7}{*}{ Tomato } & \multirow{7}{*}{$\begin{array}{l}\text { Helicoverpa } \\
\text { armigera }\end{array}$} & & & & Bacillus subtilis & GU391355 \\
\hline & & & & & & & Pseudomonas sp. & JF266598 \\
\hline & & & & & & & Bacillus $s p$ & HQ651056 \\
\hline & & & & & & & Acinetobacter sp. & HM629807 \\
\hline & & & & & & & Ochrobactrum sp. & HM629806 \\
\hline & & & & & & & $\begin{array}{l}\text { Stenotrophomonas } \\
\text { maltophilia }\end{array}$ & HM629805 \\
\hline & & & & & & & Pseudomonas sp. & HM629804 \\
\hline \multirow[t]{2}{*}{24} & \multirow[t]{2}{*}{ T. chilonis } & \multirow[t]{2}{*}{ Cotton } & \multirow[t]{2}{*}{$\begin{array}{l}\text { Helicoverpa } \\
\text { armigera }\end{array}$} & \multirow[t]{2}{*}{ Davanagere } & \multirow[t]{2}{*}{ Karnataka } & \multirow[t]{2}{*}{$\begin{array}{l}14.467^{\circ} \mathrm{N} \\
75.924^{\circ} \mathrm{E}\end{array}$} & $\begin{array}{l}\text { Stenotrophomonas } \\
\text { sp. }\end{array}$ & JF266601 \\
\hline & & & & & & & $\begin{array}{l}\text { Stenotrophomonas } \\
\text { maltophilia }\end{array}$ & JF266599 \\
\hline 25 & T. chilonis & Cabbage & $\begin{array}{l}\text { Plutella xylo- } \\
\text { stella }\end{array}$ & Pune & Maharashtra & $\begin{array}{l}18.520^{\circ} \mathrm{N} \\
73.857^{\circ} \mathrm{E}\end{array}$ & Bacillus cereus & JF736842 \\
\hline 26 & T. chilonis & Cabbage & $\begin{array}{l}\text { Plutella xylo- } \\
\text { stella }\end{array}$ & Navsari & Gujarat & $\begin{array}{l}20.949^{\circ} \mathrm{N} \\
72.914^{\circ} \mathrm{E}\end{array}$ & Bacillus cereus & JF736840 \\
\hline 27 & T. chilonis & Cabbage & $\begin{array}{l}\text { Plutella xylos- } \\
\text { tella }\end{array}$ & Ludhiana & Punjab & $\begin{array}{l}30.910^{\circ} \mathrm{N}, \\
75.850^{\circ} \mathrm{E}\end{array}$ & Bacillus cereus & JF736841 \\
\hline \multicolumn{9}{|c|}{ Locations from where no cultural microbes were obtained } \\
\hline \multirow[t]{2}{*}{28} & $\begin{array}{l}\text { T. danau- } \\
\text { sicida }\end{array}$ & Weed & $\begin{array}{l}\text { Unknown } \\
\text { eggs }\end{array}$ & Bangalore & Karnataka & $\begin{array}{l}12.967^{\circ} \mathrm{N} \\
77.567^{\circ} \mathrm{E}\end{array}$ & & \\
\hline & T. chilonis & Cotton & $\begin{array}{l}\text { Helicoverpa } \\
\text { armigera }\end{array}$ & Bangalore & Karnataka & $\begin{array}{l}12.967^{\circ} \mathrm{N} \\
77.567^{\circ} \mathrm{E}\end{array}$ & & \\
\hline 29 & T. achaeae & Castor & $\begin{array}{l}\text { Acanthodelta } \\
\text { janata }\end{array}$ & $\begin{array}{l}\text { Nelaman- } \\
\text { gala }\end{array}$ & Karnataka & $\begin{array}{l}13.500^{\circ} \mathrm{N} \\
77.230^{\circ} \mathrm{E}\end{array}$ & & \\
\hline 30 & T. chilonis & Castor & $\begin{array}{l}\text { Acanthodelta } \\
\text { janata }\end{array}$ & Jorhat & Assam & $\begin{array}{l}26.750^{\circ} \mathrm{N} \\
94.220^{\circ} \mathrm{E}\end{array}$ & & \\
\hline 31 & T. chilonis & Sugarcane & $\begin{array}{l}\text { Chilo auri- } \\
\text { cilius }\end{array}$ & Jalandhar & Punjab & $\begin{array}{l}31.326^{\circ} \mathrm{N} \\
75.576^{\circ} \mathrm{E}\end{array}$ & & \\
\hline 32 & T. chilonis & Pomegranate & $\begin{array}{l}\text { Unknown } \\
\text { eggs }\end{array}$ & Srinagar & $\begin{array}{l}\text { Jammu \& } \\
\text { Kashmir } \\
\end{array}$ & $\begin{array}{l}34.090^{\circ} \mathrm{N}, \\
74.790^{\circ} \mathrm{E}\end{array}$ & & \\
\hline
\end{tabular}

Table 2. Composition of yeast and bacteria microbiota obtained of parasitoid, Trichogramma chilonis from different host-plants and host-insects

\begin{tabular}{|l|l|l|}
\hline Microbiota diversity & Host-plant & Host-insect \\
\hline Yeast microbiota & Tomato, sugarcane & Helicoverpa armigera, Chilo auricilius, C. infuscatellus \\
\hline Candida apicola & Sugarcane & C. infuscatellus \\
\hline Hanseniaspora uvarum & Sugarcane & C. infuscatellus \\
\hline Kodamaea ohmeri & $\begin{array}{l}\text { Tomato, Sugarcane, } \\
\text { Maize }\end{array}$ & Helicoverpa armigera, Chilo auricilius, C. partellus \\
\hline Metschnikowia reukaufii & Rose & Helicoverpa armigera, unknown eggs \\
\hline Pichia anomala &
\end{tabular}


JALALI et al.

\begin{tabular}{|l|l|l|}
\hline Wickerhamomyces anomalus & Tomato, Sugarcane & $\begin{array}{l}\text { Helicoverpa armigera, Chilo auricilius, C. infuscatellus, } \\
\text { unknown eggs }\end{array}$ \\
\hline Wickerhamomyces sp. & Sugarcane & C. infuscatellus \\
\hline Zygosaccharomyces pseudorouxii & Tomato & Helicoverpa armigera \\
\hline Zygosaccharomyces rouxii & Tomato, sugarcane & Helicoverpa armigera, . infuscatellus \\
\hline Bacterial microbiota & Tomato & Helicoverpa armigera \\
\hline Acinetobacter sp. & Cabbage & Plutella xylostella \\
\hline Bacillus cereus & Tomato & Helicoverpa armigera \\
\hline Bacillus sp & Tomato & Helicoverpa armigera \\
\hline Bacillus subtilis & Tomato & Helicoverpa armigera \\
\hline Ochrobactrum sp. & Tomato & Helicoverpa armigera \\
\hline Pseudomonas sp. & Tomato & Helicoverpa armigera \\
\hline Stenotrophomonas maltophilia & Cotton & Helicoverpa armigera \\
\hline Stenotrophomonas sp. &
\end{tabular}

Zygosaccharomyces rouxii was obtained only from Tamil Nadu and Karnataka (southern states) that too only from Sugarcane and tomato. Zygosaccharomyces pseudorouxii has been observed only in samples from Tamil Nadu.

All 33 cultures of yeast recorded from different host-plants belonged to class Saccharomycetes, whereas in bacteria from different host-plants belonged to the class Bacilli (42.85\%), Gammaproteobacteria (50.0\%) and Alphaproteobacteria (7.14\%) of diversity. The class Saccharomycetes was recorded from seven host-insects, viz., Helicoverpa armigera, Chilo auricilius, C. infuscatellus, C. partellus, Acanthodelta janata, Plutella xylostella and unknown insect eggs. The Class Bacilli was recorded from Plutella xylostella and Helicoverpa armigera, Class Gammaproteobacteria from Plutella xylostella and Helicoverpa armigera and Class Alphaproteobacteria from Helicoverpa armigera only. Bacillus cereus was observed only in specimens from Plutella xylostella cabbage field and not from specimens from Helicoverpa on cotton.

A total of 33 yeast ITS sequences were used for the taxonomic identification. The taxonomic composition of the Fungi is shown in Supplementary Table 1. Prediction Confidence threshold is given in brackets. All the ITS sequences of Candida cf. apicola (4), Kodamaea ohmeri (2), Pichia anomala (2), Wickerhamomyces anomalus (12), Zygosaccharomyces rouxii (3) species correctly identified to corresponding species. In contrast, there were a total of five sequences of Metschnikowia reukaufii isolate and Zygosaccharomyces pseudorouxii (2) were matched with the Metschnikowia_cibodasensis and Zygosaccharomyces_rouxii species/, whereas Metschnikowia reukaufii isolate TCE2 is showed close association with another genus Naumovozyma. Also, Hanseniaspora uvarum strain Tcy19 (HM601459) sequence could not match with same species.

A total of 13 Bacterial 16s rDNA sequences were used for the taxonomic identification. The taxonomic composition of the Bacteria is shown in Supplementary Table 2. Prediction Confidence threshold is given in brackets. Our results revealed that there were total 6 sequences represented within the Bacillus genus. Among the most abundant genera were Stenotrophomonas (3), Pseudomonas (2), Acinetobacter (1), and Ochrobactrum (1).

In the insects, their diversity and ecological niche they occupy makes it difficult to generalize microbiota associated with them. The microbiota generally varies with feeding habits, like sap feeders (sucking pests) to termites (wood or cellulose feeder) to insects which feed on foliage or tissue / fruit borers (Rizzi et al., 2013). Another group of insects, parasitoids, which essentially are surviving on its host and as a free-living adults feed on fruits, flowers and nectar, acquire generally yeast and sometimes bacterial microbiota (Suh et al., 2004; Nguyen et al., 2006; Srinatha et al., 2015). In insects, microbiota-insect host-plant-host interactions are formed by different factors because insects lack a 'classical' adaptive protected system (McFall-Ngai, 2007). Colman et al. (2012) observed that it is one of the reasons that some insects contain fewer microbiota communities. In the present study, most interesting aspect was that at least three yeast species obtained from parasitoid, T. chilonis, viz., Wickerhamomyces sp., Kodamaea ohmeri and Hanseniasopra uvarum were recorded from Chilo infuscatellus on sugarcane but completely absent from any other host-plant, which suggests that these microbiota could be involved in the host-plant association. In contrast to yeast, 6 bacteria from parasitoid, $T$. chilonis were found associated with host-insect, $H$. armigera on tomato and B. cereus was recorded from host-insect, P. xylostella on cabbage. In an earlier study, Medina et al. (2011) also observed that association of bacteria with their host confers the trait, which allows them to differentially exploit distinct host-plants. The studies shown earlier that there are documented proof that such traits are involved in different roles such as nutritional advantages (Bernays and Klein, 
2002; Engel et al., 2012), defenses against natural enemies (Oliver et al., 2005) and enhancing biological traits in insect natural enemies (Srinatha et al., 2015; Hagen et al., 1970). It may be inferred that in similar parasitoids recorded from different host-insects, an association of yeast or bacteria may be caused by different host-plant species (Hansen and Moran, 2014). It appears that most of these species may have been transferred to parasitoid through their host-insects and hostplants, which is in agreement with Priya et al. (2012), who reported a host-plant induced variation in gut bacteria of $H$. armigera.

In the present study, wolbachia was not recorded from any of 53 populations of Trichogramma species collected from all over India. No primary bacterial or yeast species were identified in $T$. chilonis collected from any host-insect or any host-plant, which is in agreement with the previous reports (Medina et al., 2011; Priya et al., 2012). All microbiota associated with $T$. chilonis have been found associated with other insects. Of all microbes recorded on parasitoid, the most abundant crop was tomato and H. armigera was the host-insect on which $48 \%$ of diversity was recorded. Wickerhamomyces anomalus and M. reukaufii were the most prominent of all microbial diversity in the present study.

Host associated demarcation may be defined as the presence of genetically different, host associated populations have been studied earlier for the microbes associated with the insect hosts. Microbial symbionts, especially of herbivore insects, help in allowing the insect species to choose diverse plant species (Vorwerk et al., 2007; Leonardo and Muiru, 2003; Simon et al., 2003; Tsuchida et al., 2004; Ferrari et al., 2007). Some bacterial symbionts are known to develop the immune system of insects (de Souza et al., 2009).

Some species of symbionts may be present in higher population due to their occurrence on the floral parts of the host plants on which the insects obtain nectar as food. Though some species of symbionts may occur in low percentage of samples they may play an important role by providing specific amino acids to the insect. The differences in the species isolated from insects collected from different host-plants may be explained as allochronic isolations. Allochronic species do not occur simultaneously in same geological time, vice-versa also may be true that is in alliance of insect population with other plant species may be dependent on the suitable symbionts harbored by them.

Microbial symbionts in insect guts help in regulating host metabolism and they provide efficient digestion of food, extraction of maximum energy from ingested food besides protecting the host from other potentially harmful pathogens. In the sucking pests like aphids, thrips and whitefly the sym- bionts may interfere with the transmission of plant viruses due to the interference in the multiplication of viruses inside the insect gut, while in egg parasitoids, which harbor symbionts, may be helped in getting better biological fitness, enhanced fecundity, more female population, insecticide resistance, etc.

In conclusion, T. chilonis, most important egg parasitoid was recorded as most dominant parasitoid in India. It harbored vast diversity of microbiota, both yeast and bacteria from different host-insects and host-plants. The yeast, Wickerhamomyces anamalus was dominant yeast species isolated, while Bacillus cereus, Pseudomonas sp. and Stenotrophomonas maltophilia were most frequently recorded bacteria species. The taxonomic identification using RDP classifier correctly identified in the respective species with high higher confidence threshold percentage ( $>80)$ support. Results revealed that most of the species among yeast representing 9 species belong to class Saccharomycetes. Bacterial taxonomic analysis suggested that major seqeunces representing Bacillus species.

\section{ACKNOWLEDGEMENTS}

Authors are thankful to the Director, ICAR-National Bureau of Agricultural Insect Resources, Bangalore, India, for providing necessary facilities for experiments and funds for this research. Authors thank Dr. H. Nagaraja, a renowned Trichogramma taxonomist, for identification of all egg parasitoids collected. High Performance Computing Facility through a network project on 'Agricultural Bioinformatics and Computational Biology' at ICAR-NBAIR, Bangalore, is gratefully acknowledged for data analysis. Further, there is no conflict of interest amongst the authors.

\section{REFERENCES}

Bernays EA, Klein BA. 2002. Quantifying the symbiont contribution to essential amino acids in aphids: The importance of tryptophan for Uroleucon ambrosiae. Physiol Entomol. 27: 275-284. Crossref.

Bignell DE. 1984. The arthropod gut as an environment for microorganisms. In: Anderson JM, Rayner ADAM, Walton Exeter DWH (Eds.) Invertebrate-microbial interactions. Cambridge University Press, Cambridge, UK, pp. 205-227.

Bilodeau E, Guay J-F, Turgeon J, Cloutier C. 2013. Survival to parasitoids in an insect hosting defensive symbionts: A multivariate approach to polymorphic traits affecting host use by its natural enemy. PLoS One. 8: e60708. Crossref. PMid:23565269 PMCid:PMC3614562 
Bourtzis K, Miller T. 2003. Insect symbiosis. CRC Press, Boca Raton, FL, USA, pp. 324. Crossref. PMCid:PMC1462605

Broderick NA, Raffa KF, Handelsman J. 2006. Midgut bacteria required for Bacillus thuringiensis insecticidal activity. Proc Natl Acad Sci USA. 103: 15196-15199. Crossref. PMid:17005725 PMCid:PMC1622799

Chandler JA, Morgan Lang J, Bhatnagar S, Eisen JA, Kopp A. 2011. Bacterial communities of diverse Drosophila species: Ecological context of a host-microbe model system. PLoS Genet. 7: e1002272. Crossref. PMid:21966276 PMCid:PMC3178584

Colman DR, Toolson EC, Takacs-Vesbach CD. 2012. Do diet and taxonomy influence insect gut bacterial communities? Mol Ecol. 21: 5124-5137. Crossref. PMid:22978555

de Souza DJ, Bézier A, Depoix D, Drezen JM, Lenoir A. 2009. Blochinannia endosymbionts improve colony growth and immune defence in the ant Camponotus fellah. BMC Microbiol. 9: 29. Crossref. PMid:19200360 PMCid:PMC2660346

Deshpande V, Wang Q, Greefield P, Charleston M, PorrasAlfaro A, Kuske CR, Cole JR, Midgley DJ, Tran-Dinh N. 2016. Fungal identification using a Bayesian classifier and the 'Warcup' training set of internal transcribed spacer sequences. Mycologia 108: 1-5. Crossref. PMid:26553774

Dillon RJ, Dillon VM. 2004. The gut bacteria of insects: Non pathogenic interactions. Annu Rev Entomol. 49: 71-92. Crossref. PMid:14651457

Engel P, Martinsonb VG, Moran NA. 2012. Functional diversity within the simple gut microbiota of the honey bee. Proc Natl Acad Sci USA. 109: 11002-11007. Crossref. PMid:22711827 PMCid:PMC3390884

Ferrari J, Godfray HC, Faulconbridge AS, Via S. 2006. Population differentiation and genetic variation in host choice among PEA aphids from eight host plant genera. Evolution. 60: 1574-1584. Crossref. PMid:17017058

Ferrari J, Scarborough CL, Godfray HCJ. 2007. Genetic variation in the effect of a facultative symbiont on host-plant use by pea aphids. Oecologia 153: 323-329. Crossref. PMid:17415589
Ferrari J, Vavre F. 2011. Bacterial symbionts in insects or the story of communities affecting communities. Philos Trans R Soc Lond B Biol Sci. 366: 1389-1400. Crossref. PMid:21444313 PMCid:PMC3081568

Gauthier J-P, Outreman Y, Mieuzet L, Simon J-C. 2015. Bacterial communities associated with host-adapted populations of pea aphids revealed by deep sequencing of 16S ribosomal DNA. PLoS One 10: e0120664. Crossref. PMid:25807173 PMCid:PMC4373712

Hagen KS, Tassan RL, Sawall Jr EF. 1970. Some ecophysiological relationships between certain Chrysopa, honeydews and yeasts. Boll Lab Entomol Agrar Filippo Silvestri Portici. 28: 113-134.

Hammer TJ, Dickerson JC, Fierer N. 2015. Evidence-based recommendations on storing and handling specimens for analyses of insect microbiota. Peer J. 18: 3e1190.

Hansen AK, Moran NA. 2014. The impact of microbial symbionts on host plant utilization by herbivorous insects. Mol Ecol. 23: 1473-1496. Crossref. PMid:23952067

Hassan SA. 1997. Selection of species of Trichogramma for use in biological control programs. In: (Parra JRP, Zucchi RA (eds.) Trichogramma and Applied Biological Control, Piracicaba: ESALQ, Brazil, pp. 183-205. PMid:9272632

Jalali SK, Singh SP. 1993. Superior strain selection of the egg parasitoid Trichogramma chilonis Ishii - Biological parameters. J Biol Control 7: 57-60.

Jalali SK, Singh SP, Venkatesan T, Murthy KS, Lalitha Y. 2006. Development of endosulfan tolerant strain of an egg parasitoid Trichogramma chilonis Ishii (Hymenoptera: Trichogrammatidae). Indian J Exp Biol. 44: 584-590. PMid:16872050

Kaufman MG, Klug MJ. 199.) The contribution of hindgut, bacteria to dietary carbohydrate utilization by crickets (Orthoptera: Gryllidae). Comp Biochem Physiol. 98: 117-123. Crossref.

Lachance MA, Bowles JM. 2002. Metschnikowia arizonensis and Metschnikowia dekortorum, two new large-spore yeast species associated with floricolous beetles. FEMS Yeast Res. 2: 81-86. Crossref.

Leonardo TE, Muiru GT. 2003. Facultative symbionts are associated with host plant specialization in pea aphid 
Host-insect and host-plant associated diversity in microbiota isolated from most important Oriental-Australian region egg parasitoid

populations. Proc R Soc Lond B Biol Sci. 270: 209-212.

Crossref. PMid:14667385 PMCid:PMC1809968

McFall-Ngai MJ. 2007. Adaptive immunity: care for the community. Nature 445: 153. Crossref. PMid: 17215830

Medina FF, Nachappa P, Tamborindeguy C. 2011. Differences in bacterial diversity of host-associated populations of Phyllexera notabilis Pergande (Hemiptrea: Phylloxeridae) in pecan and water hickory. $J$ Evol Biol. 24: 761-771. Crossref. PMid:21261774

Meyer JM, Hoy MA. 2008. Molecular survey of endosymbionts in Florida populations of Diaphorina citri (Hemiptera: Psyllidae) and its parasitoids Tamarixia radiata (Hymenoptera: Eulophidae) and Diaphorencyrtus aligarhensis (Hymenoptrea: Encyrtidae). Florida Entomol. 91: 294-304. Crossref.

Montagna M, Chouaia B, Mazza G, Prosdocimi EM, Crotti E, Mereghetti V, Vacchini V, Giorgi A, Biase AD, Cervo R, Lozzia GC, Alma A, Bandi C, Daffonchio D. 2015. Effects of the diet on the microbiota of the red palm weevil (Coleoptera: Dryophthoridae). PLoS One 10: e0117439. Crossref. PMid:25635833 PMCid:PMC4311986

Mrázek J, Strosová L, Fliegerová K, Kott T, Kopencý J. 2008. Diversity of insect intestinal microflora. Folia Microbiol. 53: 229-233. Crossref. PMid: 18661298

Nagarkatti S, Nagaraja H. 1978. Experimental comparison of laboratory reared vs. wild-type Trichogramma confusum (Hym.: Trichogrammatidae) I. Fertility, fecundity and longevity. BioControl 23: 129-136.

Ngo CT, Aujoulat F, Veas F, Jumas-Bilak E, Manguin S. 2015. Bacterial diversity associated with wild caught Anopheles mosquitoes from Dak Nong Province, Vietnam using culture and DNA Fingerprint. PLoS One 10: e0118634. Crossref. PMid:25747513 PMCid:PMC4352016

Nguyen NH, Suh SO, Erbil CK, Blackwell M. 2006. Metschnikowia noctiluminum sp. nov., Metschnikowia corniflorae sp. nov., and Candida chrysomelidae sp. nov., isolated from green lacewings and beetles. Mycol Res. 110: 346-356. Crossref. PMid: 16483756

Oliver KM, Moran RL, Hunter MS. 2005. Variation in resistance to parasitism in aphids is due to symbionts not host genotype. Proc Natl Acad Sci USA. 102: 12795-12800. Crossref. PMid:16120675 PMCid:PMC1200300

Priya NG, Ojha A, Kajla MK, Raj A, Raman R. 2012. Host plant induced variation in gut bacteria of
Helicoverpa armigera. PLoS One 7, e30768. Crossref. PMid:22292034 PMCid:PMC3266921

Rizzi A, Crotti E, Borruso L, Jucker C, Lupi D, Colombo M, Daffonchio D. 2013. Characterization of the bacterial community associated with larvae and adults of Anoplophora chinensis collected in Italy by culture and culture-independent methods. BioMed Res Int. 2013: 420287. Crossref. PMid:24069601 PMCid:PMC3771249

Simon JC, Carré S, Boutin M, Prunier-Leterme N, SabaterMunoz, Latorre A, Bournoville R. 2003. Host Based divergence in populations of the pea aphid: insights from nuclear markers and the prevalence of facultative symbionts. Proc Biol Sci. 270: 1703-1712. Crossref. PMid:12964998 PMCid:PMC1691435

Srinatha HS, Jalali SK, Sriram S, Chakravarthy AK. 2015. Isolation of microbes associated with field-collected populations of the egg parasitoid, Trichogramma chilonis capable of enhancing biotic fitness. Biocontrol Sci Technol. 25: 789-802. Crossref.

Stireman JO, Nason JD, Heard SB. 2005. Host-associated genetic differentiation in phytophagous insects: General phenomenon or isolated exceptions? Evidence from a goldenrod insect community. Evolution. 59: 2573-2587. Crossref. PMid:16526505

Suh SO, Gibson CM, Blackwell M. 2004. Metschnikowia chrysoperlae sp. nov., Candida picachoensis sp. nov. and Candida pimensis sp. nov., isolated from the green lacewings Chrysoperla comanche and Chrysoperla carnea (Neuroptera: Chrysopidae). Int J Syst Evol Microbiol. 54: 1883-1890. Crossref. PMid:15388758

Tagami Y, Doi M, Sugiyama K, Tatara A, Saito T. 2006. Survey of leafminers and their parasitoids to find endosymbionts for improvement of biological control. Biol Control. 38: 210-216. Crossref.

Toju H, Fukatsu T. 2011. Diversity and infection prevalence of endosymbionts in natural populations of the chestnut weevil: relevance of local climate and host plants. $\mathrm{Mol}$ Ecol. 20: 853-868. Crossref. PMid:21199036

Tsuchida T, Koga R, Fukatsu T. 2004. Host plant specialization governed by facultative symbiont. Science 303: 1989. Crossref. PMid: 15044797

Vorwerk S, Martinez-Torres D, Forneck A. 2007. Pantoea agglomerans-associated bacteria in grape phylloxera 
JALALI et al.

(Daktulosphaira vitifoliae, Fitch). Agric Forest Entomol. 9: 57-64. Crossref.

Wang Q, Garrity GM, Tiedje JM, Cole JR. 2007. Naïve Bayesian classifier for rapid assignment of rRNA sequences into the new bacterial taxonomy. Appl Environ Microbiol. 73: 5261-5267. Crossref. PMid:17586664 PMCid:PMC1950982

White TJ, Bruns TD, Lee SB, Taylor JW. 1990. Amplification and direct sequencing of fungal ribosomal RNA genes for phylogenetics. In: Innis MA, Gelfand DH, Sninsky JJ, White TJ (Eds.) PCR Protocols: A guide to Methods and Applications. Academic Press: San Diego, U.S.A., 1990, pp. 315-322. Crossref.

Yun JH, Roh SW, Whon TW, Jung M-J, Kim MS, Park D-S, Yoon C, Nam Y-D, Kim Y-J, Choi J-H, Kim J-Y, Shin N-R, Kim S-H, Lee W-J, Bae J-W. 2014. Insect gut bacterial diversity determined by environmental habitat, diet, developmental stage, and phylogeny of host. Appl Environ Microbiol. 80: 5254-5264. Crossref. 